\title{
Significant Stabilization
}

National Cancer Institute

\section{Source}

National Cancer Institute. Significant Stabilization. NCI Thesaurus. Code C54017.

Showing important indications that a patient is nearing a condition of little change. 